been suggested that progression of the pathology in $\mathrm{AD}$ is related to the connections between the areas displaying early deposits. This finding might aid in furthering our understanding of the generation, deposition and clearance of amyloid deposits in Alzheimer disease.
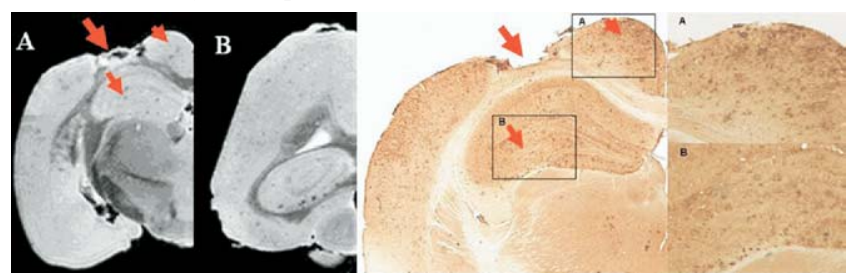

IC-P-003 HIPPOCAMPAL AND WHITE MATTER CHANGES IN MCI PATIENTS ACCORDING TO THE PRESENCE OR THE ABSENCE OF SUBCORTICAL VASCULAR CHANGES

Yong Soo Shim, Dong Won Yang, Young-Min Shon, Bora Yoon, Beum-Saeng Kim, College of Medicine, The Catholic University of Korea, Seoul, Republic of Korea. Contacte-mail: ysshim@catholic.ac.kr

Background: Mild cognitive impairment (MCI) refers to a transitional state between normal aging and dementia, and it is clinically heterogeneous. Although most imaging studies of MCI have focused on gray matter alterations, many MCI patients have subcortical vascular disease on magnetic resonance imaging (MRI) scan. However it is not known whether these findings are associated with the cognitive dysfunction. Diffusion tensor imaging (DTI) detects the microstructural alterations in white matter by measuring the directionality of molecular diffusion. Objective: We investigated the alterations of white matter as well as hippocampus in MCI patients according to the presence or the absence of subcortical vascular disease by using DTI, and compared the differences between two groups. Methods: Forty consecutive patients with memory complaints, at least one neuropsychological memory test below 1.5 standard deviation of the normal for age and education, and maintained activities of daily living, were included. 21 patients with MCI had no ischemic lesions and 19 patients were found to have subcortical vascular changes, by the Erkinjuntti's imaging criteria. For 21 non-vascular MCI (nvMVI), 19 vascular MCI (vMCI) and 17 controls, mean diffusivity (MD) and fractional anisotropy (FA) were measured in the bilateral temporal, frontal, parietal and occipital white matter regions as well as in the bilateral hippocampi, sentrum semiovale and the corpus callosum (genu and splenum), and the differences were compared by analysis of variance and multiple comparison. Results: All patients with MCI, both vascular and non-vascular, showed that decreased FA and increased MD values in the other regions except occipital white matter, compared to the controls. For the FA, both types of MCI patients had decreased FA values in the corpus callosum, frontal and temporal regions, compared to the controls. In the parietal areas and centrum semiovale, vMCI patients had more decreased FA values than nvMCI patients and the controls. In the hippocampus, FA values were lowest in the nvMCI patients. FA values of vMCI patients were also significantly lower than the controls. The findings of region-specific MD increases were similar to those of FA. Conclusions: The DTI can be a useful tool to quantify MCI pathology in vivo, and to evaluate the alterations of intracerebral microstructure.

Table 1

Demographic Data

\begin{tabular}{lllrr}
\hline & $\begin{array}{l}\text { Normal } \\
(\mathbf{n = 1 7})\end{array}$ & $\begin{array}{l}\text { nvMCI } \\
(\mathbf{n = 2 1})\end{array}$ & \multicolumn{1}{l}{$\begin{array}{l}\text { vMCI } \\
(\mathbf{n = 1 9 )}\end{array}$} & p Value \\
\hline Age & $68.76 \pm 3.61$ & $72.95 \pm 6.90$ & $70.32 \pm 5.88$ & 0.083 \\
K-MMSE & $28.73 \pm 1.10$ & $24.50 \pm 2.53$ & $25.28 \pm 2.97$ & $<0.001$ \\
HIS & $0.00 \pm 0.00$ & $2.19 \pm 1.94$ & $6.16 \pm 2.65$ & $<0.001$ \\
PVHI & $2.59 \pm 1.91$ & $3.95 \pm 2.11$ & $7.11 \pm 1.29$ & $<0.001$ \\
DWHI & $0.12 \pm 0.33$ & $0.76 \pm 1.26$ & $3.42 \pm 1.57$ & $<0.001$ \\
Lacune & $0.65 \pm 0.79$ & $0.95 \pm 1.50$ & $3.79 \pm 1.99$ & $<0.001$ \\
\hline
\end{tabular}

Table 2

Fractional Anisotropy Values of Various Regions in Both Types of MCI Patients

\begin{tabular}{|c|c|c|c|c|}
\hline & $\begin{array}{l}\text { Normal } \\
(\mathrm{n}=17)\end{array}$ & $\begin{array}{l}\text { nvMCI } \\
(\mathrm{n}=21)\end{array}$ & $\begin{array}{l}\text { vMCI } \\
(n=19)\end{array}$ & p Value \\
\hline FA-T & $\begin{array}{l}0.35 / 0.36 \\
( \pm 0.03 / 0.04)\end{array}$ & $\begin{array}{l}0.32 / 0.31 \\
( \pm 0.03 / 0.02)\end{array}$ & $\begin{array}{l}0.32 / 0.31 \\
( \pm 0.04 / 0.06)\end{array}$ & $0.004 / 0.003$ \\
\hline FA-H & $\begin{array}{l}0.15 / 0.15 \\
( \pm 0.02 / 0.01)\end{array}$ & $\begin{array}{l}0.11 / 0.11 \\
( \pm 0.01 / 0.03)\end{array}$ & $\begin{array}{l}0.13 / 0.13 \\
( \pm 0.02 / 0.02)\end{array}$ & $<0.001 /<0.001$ \\
\hline FA-O & $\begin{array}{l}0.37 / 0.37 \\
( \pm 0.03 / 0.04)\end{array}$ & $\begin{array}{l}0.34 / 0.35 \\
( \pm 0.06 / 0.05)\end{array}$ & $\begin{array}{l}0.33 / 0.33 \\
( \pm 0.07 / 0.07)\end{array}$ & $0.119 / 0.074$ \\
\hline FA-CCg & $0.72 \pm 0.06$ & $0.63 \pm 0.07$ & $0.65 \pm 0.06$ & $<0.001$ \\
\hline FA-CCs & $0.78 \pm 0.03$ & $0.69 \pm 0.09$ & $0.73 \pm 0.07$ & $<0.001$ \\
\hline FA-P & $\begin{array}{l}0.44 / 0.43 \\
( \pm 0.04 / 0.04)\end{array}$ & $\begin{array}{l}0.42 / 0.42 \\
( \pm 0.05 / 0.06)\end{array}$ & $\begin{array}{l}0.34 / 0.35 \\
( \pm 0.08 / 0.08)\end{array}$ & $<0.001 / 0.001$ \\
\hline FA-F & $\begin{array}{l}0.39 / 0.40 \\
( \pm 0.03 / 0.03)\end{array}$ & $\begin{array}{l}0.29 / 0.31 \\
( \pm 0.06 / 0.04)\end{array}$ & $\begin{array}{l}0.30 / 0.30 \\
( \pm 0.06 / 0.06)\end{array}$ & $<0.001 /<0.001$ \\
\hline FA-CS & $\begin{array}{l}0.44 / 0.45 \\
\pm 0.04 / 0.05)\end{array}$ & $\begin{array}{l}0.44 / 0.45 \\
\pm 0.03 / 0.05)\end{array}$ & $\begin{array}{l}0.36 / 0.37 \\
( \pm 0.06 / 0.06)\end{array}$ & $<0.001 /<0.001$ \\
\hline ADC-T & $\begin{array}{l}0.73 / 0.74 \\
( \pm 0.05 / 0.04)\end{array}$ & $\begin{array}{l}0.79 / 0.79 \\
( \pm 0.12 / 0.15)\end{array}$ & $\begin{array}{l}0.82 / 0.82 \\
( \pm 0.06 / 0.07)\end{array}$ & $<0.001 / 0.001$ \\
\hline ADC-H & $\begin{array}{l}0.81 / 0.82 \\
( \pm 0.06 / 0.07)\end{array}$ & $\begin{array}{l}0.92 / 0.96 \\
( \pm 0.12 / 0.15)\end{array}$ & $\begin{array}{l}1.02 / 0.98 \\
( \pm 0.14 / 0.13)\end{array}$ & $<0.001 / 0.001$ \\
\hline ADC-O & $\begin{array}{l}0.71 / 0.70 \\
( \pm 0.05 / 0.04)\end{array}$ & $\begin{array}{l}0.78 / 0.75 \\
( \pm 0.14 / 0.13)\end{array}$ & $\begin{array}{l}0.78 / 0.78 \\
( \pm 0.12 / 0.12)\end{array}$ & $0.119 / 0.111$ \\
\hline ADC-CC & $0.80 \pm 0.08$ & $0.92 \pm 0.09$ & $0.90 \pm 0.07$ & $<0.001$ \\
\hline $\mathrm{ADC}-\mathrm{CCs}$ & $0.72 \pm 0.07$ & $0.83 \pm 0.10$ & $0.81 \pm 0.11$ & 0.001 \\
\hline ADC-P & $\begin{array}{l}0.68 / 0.68 \\
( \pm 0.03 / 0.04)\end{array}$ & $\begin{array}{l}0.74 / 0.77 \\
( \pm 0.06 / 0.07)\end{array}$ & $\begin{array}{l}0.89 / 0.89 \\
( \pm 0.12 / 0.16)\end{array}$ & $<0.001 /<0.001$ \\
\hline ADC-F & $\begin{array}{l}0.73 / 0.73 \\
( \pm 0.04 / 0.05)\end{array}$ & $\begin{array}{l}0.78 / 0.78 \\
( \pm 0.05 / 0.07)\end{array}$ & $\begin{array}{l}0.86 / 0.87 \\
( \pm 0.11 / 0.09)\end{array}$ & $<0.001 /<0.001$ \\
\hline ADC-CS & $\begin{array}{l}0.66 / 0.68 \\
( \pm 0.03 / 0.04)\end{array}$ & $\begin{array}{l}0.67 / 0.68 \\
( \pm 0.03 / 0.04)\end{array}$ & $\begin{array}{l}0.82 / 0.82 \\
( \pm 0.16 / 0.16)\end{array}$ & $<0.001 /<0.001$ \\
\hline
\end{tabular}
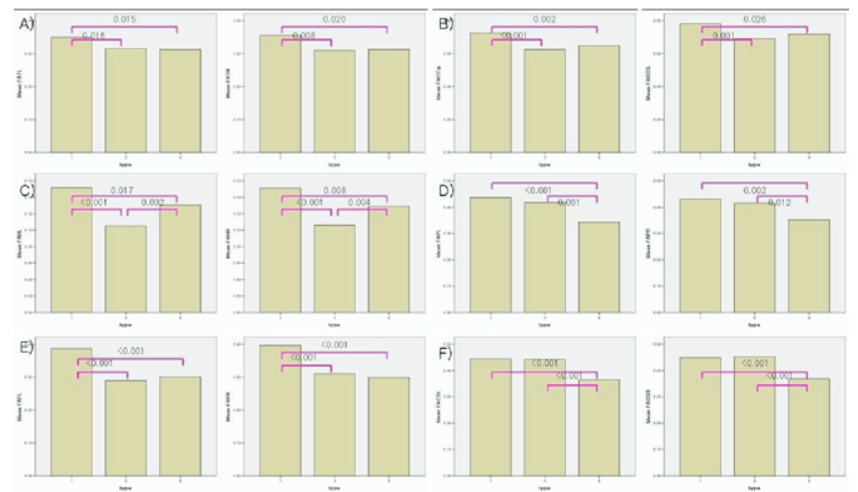

\section{IC-P-004 DISRUPTION OF DEFAULT MODE CORRELATIONS IN AGING AND ALZHEIMER'S DISEASE}

Jessica R. Andrews ${ }^{1}$, Abraham Z. Snyder ${ }^{2,3}$, Justin L. Vincent ${ }^{2}$, Cindy Lustig ${ }^{4}$, Michael D. Fox ${ }^{2,1}$, Marcus E. Raichle ${ }^{2}$, John C. Morris ${ }^{5}$, Randy L. Buckner ${ }^{6,7},{ }^{1}$ Division of Biology and Biomedical Sciences, Washington University, St. Louis, MO, USA; ${ }^{2}$ Mallinckrodt Institute of Radiology, Washington University School of Medicine, St. Louis, MO, USA; ${ }^{3}$ Department of Neurology, Washington University School of Medicine, St. Louis, MO, USA; ${ }^{4}$ Department of Psychology, University of Michigan, Ann Arbor, MI, USA; ${ }^{5}$ Alzheimer's Disease Research Center, Department of Neurology, Washington University School of Medicine, St. Louis, MO, USA; ${ }^{6}$ Department of Psychology and Center for Brain Sciences, Harvard University, Cambridge, MA, USA;

${ }^{7}$ Athinoula A. Martinos Center for Biomedical Imaging, Massachusetts General Hospital, Boston, MA, USA. Contact e-mail:

jandrews@wustl.edu

Background: Growing interest has emerged in how activity patterns engaged in default cognitive modes differ in aging and AD (Lustig et al., 2003 PNAS; Greicius et al., 2004 PNAS). Some data have even suggested default activity patterns may be causal to disease-associated processes 
(Buckner et al., $2005 \mathrm{JN}$ ). Objective(s): To further investigate these observations, the present study characterized default mode activity using analysis of low frequency functional correlations between brain regions (Biswal et al., 1995 MRM; Fox et al., 2005 PNAS). BOLD-contrast fMRI imaging was used and the correlation coefficient between regions was the dependent measure. Methods: Functional correlations were explored in a large sample of 38 young adults (age $22.4 \mathrm{y} ; 18 \mathrm{M}$ ), 55 nondemented older adults $(76.5 \mathrm{y} ; 18 \mathrm{M})$ and 17 older adults $(76.9 \mathrm{y} ; 12 \mathrm{M})$ with a clinical diagnosis of $\mathrm{AD}$ in accordance with validated criteria using standard assessment protocols for both clinical and neuropsychological measures. Data were from Lustig et al. (2003 PNAS). To minimize anatomical biases, seed regions used for correlation analyses were defined in a separate data set of an equal number of young, nondemented older, and AD participants $(\mathrm{n}=24$ total). Many participants also underwent diffusion tensor imaging (DTI) at nearby times to assess white matter integrity. Conclusions: Results revealed that functional correlations were dramatically reduced between anterior and posterior regions comprising the default mode in nondemented older adults compared to the young. Considerably more modest reductions in correlations were noted between the nondemented and $\mathrm{AD}$ older adult groups. We also examined relationships between functional correlation measures, performance on neuropsychological tests, and DTI in the same group of individuals, as they are useful to understand the interaction between potentially related changes in aging. Our results are consistent with the hypothesis that aging is associated with disconnection of distributed brain networks that show coordinated activity in young adults. Future analyses will explore whether our observed functional disconnection in aging is related to findings of white-matter degradation (e.g., O'Sullivan et al., 2000 Neurology; Head et al., 2004 CC) and also whether functional disconnection is prominent in nondemented aging in the absence of $\mathrm{AD}$.

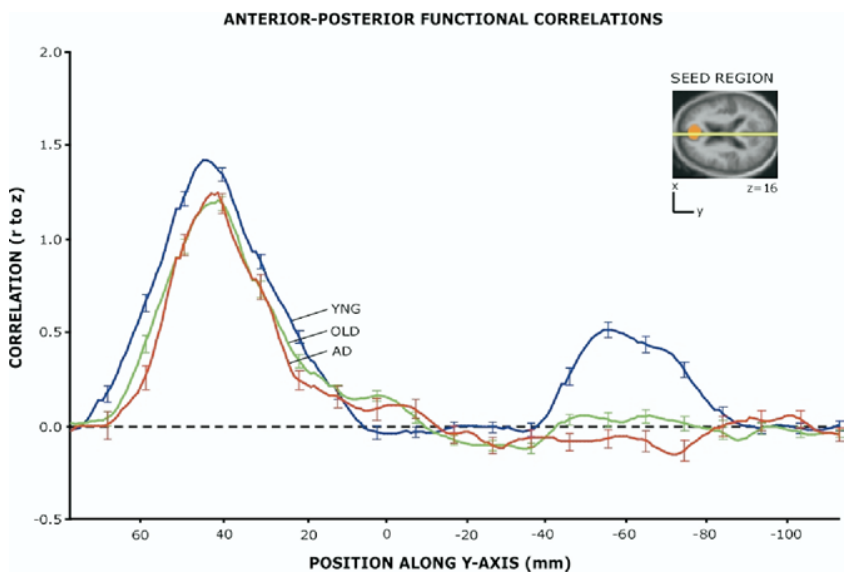

\section{IC-P-005 WHITE MATTER CHANGES ASSOCIATED WITH DEMENTIA DEVELOPMENT IN PATIENTS WITH SUBCORTICAL ISCHEMIC WHITE MATTER LESIONS: A DIFFUSION TENSOR IMAGE STUDY}

Dong Won Yang ${ }^{1}$, Beum Saeng Kim ${ }^{1}$, Young Min Shon ${ }^{1}$, Yong Soo Shim ${ }^{1}$, Hyun $\mathrm{Cho}^{1}$, Bora Yoon ${ }^{1}$, Kook-Jin $\mathrm{Ahn}^{2},{ }^{1}$ St. Mary's Hospital, Catholic University of Korea, Seoul, Republic of Korea; ${ }^{2}$ St. Mary's Hospital, Catholic University of Korea, Radiology, Seoul, Republic of Korea. Contact e-mail: neuroman@catholic.ac.kr

Background: Diffusion tensor MRI (DTI) is a new technique that may provide a better index of white matter damage in central nervous system. Both discrete lacunar infarcts and leukoaraiosis are important mechanisms in developing dementia in patients with subcortical ischemic vascular lesions. But the location and severity of white matter changes associated with development of dementia has not been established yet. Objective(s): We investigate the difference of location and severity of white matter damage in patients with subcortical ischemic vascular lesions with and without dementia. Methods: Eleven demented patients (age: $74.80 \mathrm{yrs,}$ MMSE: 22.10) who fulfill the criteria of Erkinjuntti's subcortical ischemic vascular dementia (SIVD) criteria, ten patients (age: $67.50 \mathrm{yrs}$, MMSE: 26.63) with subcortical vascular cognitive impairment with no dementia (SVCIND) and twelve healthy volunteers (age: 70.64 yrs, MMSE: 28.73) were recruited. They underwent DTI, neuropsychological assessment and clinical interview. Fractional anisotropy (FA) and mean diffusivity (MD) were measured in temporal, frontal, parietal and occipital white matter regions as well as in the corpus callosum (genu, body and splenium), cingulate white matter (anterior and posterior), internal capsule (anterior and posterior limb), superior and inferior longitudinal fasciculus, centrum semiovale, hippocampus, and anterior thalamus. Results: SVCIND and SIVD patients showed lower FA and higher MD values in the both frontal, occipital, right anterior thalamus, centrum semiovale, superior longitudinal fasciculus and in both hippocampi than the control group. Compared to SVCIND patients, SIVD patients had decreased FA values and increased MD values in both frontal areas and decreased MD in left superior longitudinal fasciculus. Conclusions: The findings support the white matter damage and disruption in the frontal region and superior association fiber is important for the cognitive dysfunctions associated with dementia development in patients with subcortical ischemic lesions. This study was supported by a grant of the Korea Health 21 R\&D Project, Ministry of Health \& Welfare, Republic of Korea (A050079).

\section{IC-P-006 ANALYSIS OF REGIONAL CEREBRAL VOLUME BETWEEN NORMAL PRESSURE HYDROCEPHALUS AND ALZHEIMER'S DISEASE}

Dong Won Yang ${ }^{1}$, Beum Saeng Kim ${ }^{1}$, Young Min Shon ${ }^{1}$, Yong Soo Shim ${ }^{1}$, Hyun Cho ${ }^{1}$, See Back Lee ${ }^{1}$, Kook-Jin Ahn ${ }^{2},{ }^{1}$ St. Mary's Hospital, Catholic University of Korea, Neurology, Seoul, Republic of Korea; ${ }^{2}$ St. Mary's Hospital, Catholic University of Korea, Radiology, Seoul, Republic of Korea. Contact e-mail:

neuroman@catholic.ac.kr

Background: Normal pressure hydrocephalus (NPH) is a reversible dementia characterized by gait disturbance, incontinence and dementia. In elderly dementia patients with dilated ventricles, it is often difficult to differentiate NPH from Alzheimer's disease (AD) with visual measurement of MRI. Objective(s): This study investigates the differences of regional cerebral volumes between NPH patients and those with AD to see if regional cerebral atrophy could be used as a distinguishing characteristic of these two disorders. Methods: MR images of 9 patients with a clinical diagnosis of NPH who improved after CSF drainage were compared with $10 \mathrm{AD}$ patients and 10 normal control volunteers. Objective, computer-aided volumetric measurement with ANALYER program of the frontal lobes, parietal lobes, occipital lobes, temporal lobes, hippocampi and ventricle were obtained. Volume of each brain regions divided with whole brain volume to correct individual variation of brain volume. Results: NPH patients and those with AD have CDR 0.5 respectively. And their MMSE score was not different statistically. Compared with those for control volunteers, NPH patients showed smaller bilateral frontal, occipital lobe and hippocampal and ventricle volumes $(\mathrm{p}<0.05)$. Compared with those for patients with $\mathrm{AD}$, patients with NPH had significantly smaller bilateral frontal, left occipital lobe and ventricle volumes $(\mathrm{p}<0.05)$. Conclusions: Clinical symptoms and signs of NPH that differ from AD may be associated with decreased volumes of bilateral frontal lobes not with hippocampal atrophy. And smaller hippocampal volumes in NPH patients may be due to mechanical compression by increased cerebrospinal fluid. Measurement of regional brain volume provides diagnostic discrimination NPH from AD. This study was supported by a grant of the Korea Health 21 R\&D Project, Ministry of Health \& Welfare, Republic of Korea (A050079). 